\title{
Pulsed ECR Source in Afterglow Operation at CERN
}

\author{
C.E. Hill, K. Langbein \\ CERN, 1211 Geneva 23, Switzerland
}

\begin{abstract}
A pulsed $14 \mathrm{GHz}$ ECR4 source, adapted to operate in the afterglow mode, was delivered by GANIL to CERN in the framework of the Heavy Ion Facility Project. After four months of operation to commission the facility, it completed its first operational run of nine weeks at the end of 1994, providing lead ions to nuclear physics and ion cooling experiments. A very stable beam, with a useful length of over $1 \mathrm{~ms}$, of $80 \mathrm{e} \mu \mathrm{A}$ of $\mathrm{Pb}^{27+}$ was provided by the source over this period. The operational problems experienced during the commissioning and operation, and the investigations to improve performance for the next experimental physics run are presented.
\end{abstract}

\section{INTRODUCTION}

CERN's original proton linac, commissioned in 1958, was shut down in 1992 after completion of the final light ion period with sulphur ions. It was immediately dismantled and construction started, on the site, of a new heavy ion linac (Linac3) intended initially for the acceleration of lead ions ${ }^{1}$. Components for this machine were provided within the framework of an international collaboration involving GANIL, Caen (ion source); INFN, Legnaro (low energy beam transport and RFQ); GSI, Darmstadt (Interdigital-H linac and some RF systems); INFN, Torino (high energy transport and filtering); IAP, Frankfurt (debuncher), and with assistance from Sweden, Switzerland, the Czech Republic and India. In June 1994 the first beam was passed to the next accelerator in the injector chain, (the booster synchrotron) and in October beam was passed to the physics experiments for a nine week operational period.

The ion source chosen for the project was of the ECR type. Normally the synchrotrons of CERN require a beam length of $400-600 \mu$ s at a repetition rate of about $1 \mathrm{~Hz}$. Although the ECR is not well suited to short pulse operation, the afterglow phenomenon can be exploited to give interesting intensities of ions with high charge states as had been demonstrated for sulphur ions ${ }^{2}$. In the optimization of the design of the new facility the output energy of the linac was defined to be $4.2 \mathrm{MeV} / \mathrm{u}$ for $\mathrm{Pb}^{53+}$. As this charge state is beyond the reach of normal ECR sources, stripping at the end of the linac was necessary. Further design optimizations indicated that at least $80 \mathrm{e} \mu \mathrm{A}$ of $\mathrm{Pb}^{25+}$ would be required from the source. In fact, to limit the X-ray emission from the linac cavities which were designed for $25+$, the $27+$ charge state was chosen for operational purposes.

GANIL carried out a series of tests on their ECR4 source and demonstrated that enhanced intensities of highly charge lead ions could indeed be obtained in the 
afterglow mode of operation which satisfied the criteria of:- a) intensity $>80$ e $\mu \mathrm{A} ; \mathrm{b}$ ) adequate useful beam length; c) pulse to pulse stability; d) emittance.

\section{THE ION SOURCE}

The ion source, shown in Fig.1, is an ECR4 ${ }^{3}$ type with an operational frequency of $14.5 \mathrm{GHz}$ and a cooled plasma chamber. Microwave power is injected into the plasma by a tuned waveguide to co-axial transition. The maximum available RF power is $2 \mathrm{~kW}$. Although designed for c.w. operation, the source can be optimized for normal pulse operation or afterglow. It uses oxygen as the plasma support gas. For lead operation, the sample material (isotopically pure ${ }^{208} \mathrm{~Pb}$ ) is evaporated into the plasma from a micro oven inserted near the plasma end of the microwave co-axial line.

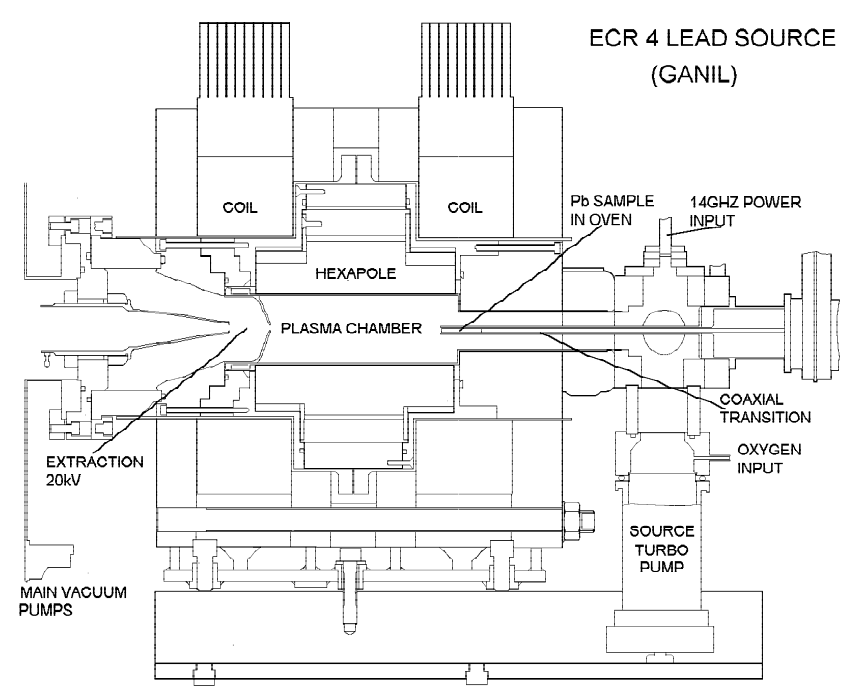

FIG. 1 Schematic of the ECR4 lead source

With the source adjusted for afterglow operation ${ }^{4}$, the discharge has a length of $50 \mathrm{~ms}$. The source is pulsed at $10 \mathrm{~Hz}$ in spite of the linac pulsing at $1 \mathrm{~Hz}$. This higher frequency is necessary to maintain the stability of the source. RF losses in the co-axial feed contribute to the heating of the sample and $5 \mathrm{~Hz}$ operation is possible with increased oven heating. Ions are extracted from the plasma, through a simple one gap extraction system immersed in the end field of the source, at $2.5 \mathrm{keV} / \mathrm{u}$ which is the injection energy of the RFQ. This gives an extraction voltage of about $20 \mathrm{kV}$, well within the insulation limit of the system.

Figure 2 shows the layout of the injector. The extracted beam from the source is analyzed in the spectrometer and measured in the Faraday cup at its outlet.

\section{SOURCE COMMISSIONING}

After delivery, installation and alignment of the source, the first plasma was 
proved necessary, especially with the magnetic field at the top of the anticipated field range. For a large part of this formation argon was used in the source to clean up the electrodes. The spectrometer was not available for use until July 1993 when an argon beam was seen in the Faraday cup. A long time was spent mastering the afterglow

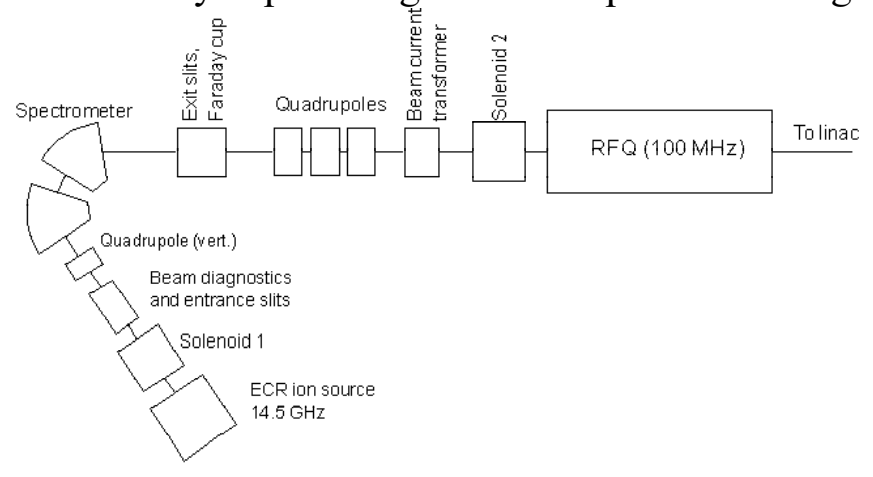

FIG. 2 Linac3 injector layout

mode of operation. Ion beams of various argon charge states were produced (at $20 \mathrm{kV}$ extraction) up to $\mathrm{Ar}^{13+}$. Testing of the spectrometer used beams of natural krypton $\left({ }^{84} \mathrm{Kr}^{15+}, 25 \mathrm{e} \mu \mathrm{A}\right)$ and xenon $\left({ }^{132} \mathrm{Xe}^{26+}, 11 \mathrm{e} \mu \mathrm{A}\right)$ and demonstrated the resolution of the spectrometer $\left(10^{-4}\right)$.

In August 1993, the source provided a lead beam for the first time and $65 \mathrm{e} \mu \mathrm{A}$ of $\mathrm{Pb}^{27+}$ with a usable beam length of $600 \mu$ s was quickly achieved but limited by vacuum losses in the transport line. This setting, referred to as the "GANIL" mode as it used settings devised by them, had the currents in the source mirror coils in the region of 1050 A (Fig. 3a). Further optimization of this beam resulted in a maximum current of about $100 \mathrm{e} \mu \mathrm{A}$.

Although not sufficiently stable in amplitude and length, very high pulses of current could be obtained by running the rear mirror coil at high currents (around 1100 A) . This mode indicated that improvements were possible and that the beam might be suitable for some special applications.

During a search of the source parameter space, a new operating point was found. At lower magnetic fields ( $900 \mathrm{~A})$, the afterglow pulse became very smooth and stable with decay tail of several milliseconds. The pulse rise time was of the order of $500 \mu \mathrm{s}$ and the plateau acceptable (Fig. 3b). Although the intensities were somewhat lower than the GANIL mode, a compromise could be found which retained the extreme stability on a pulse to pulse basis whilst keeping a good plateau (Fig. 3c). This stability proved to be invaluable in the setting up of the following accelerators. A reproducible operating current of around $80 \mathrm{e} \mu \mathrm{A}$ was adopted as standard.

One unexpected problem which arose was that the horizontal position of the beam at the entrance of the RFQ varied during the pulse. This was traced to a variation of energy in the beam from the source. During the afterglow, the total current leaving the source increased dramatically and the extraction power supply was unable

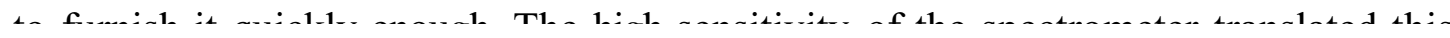


into horizontal steering. The problem was overcome by dynamically stabilizing the high voltage during the afterglow.

Heavy demands were made on the lead beam once it became available for the commissioning of the new machine (Linac3) and for the subsequent accelerators. Effectively, the beam was used from May to September for this purpose.
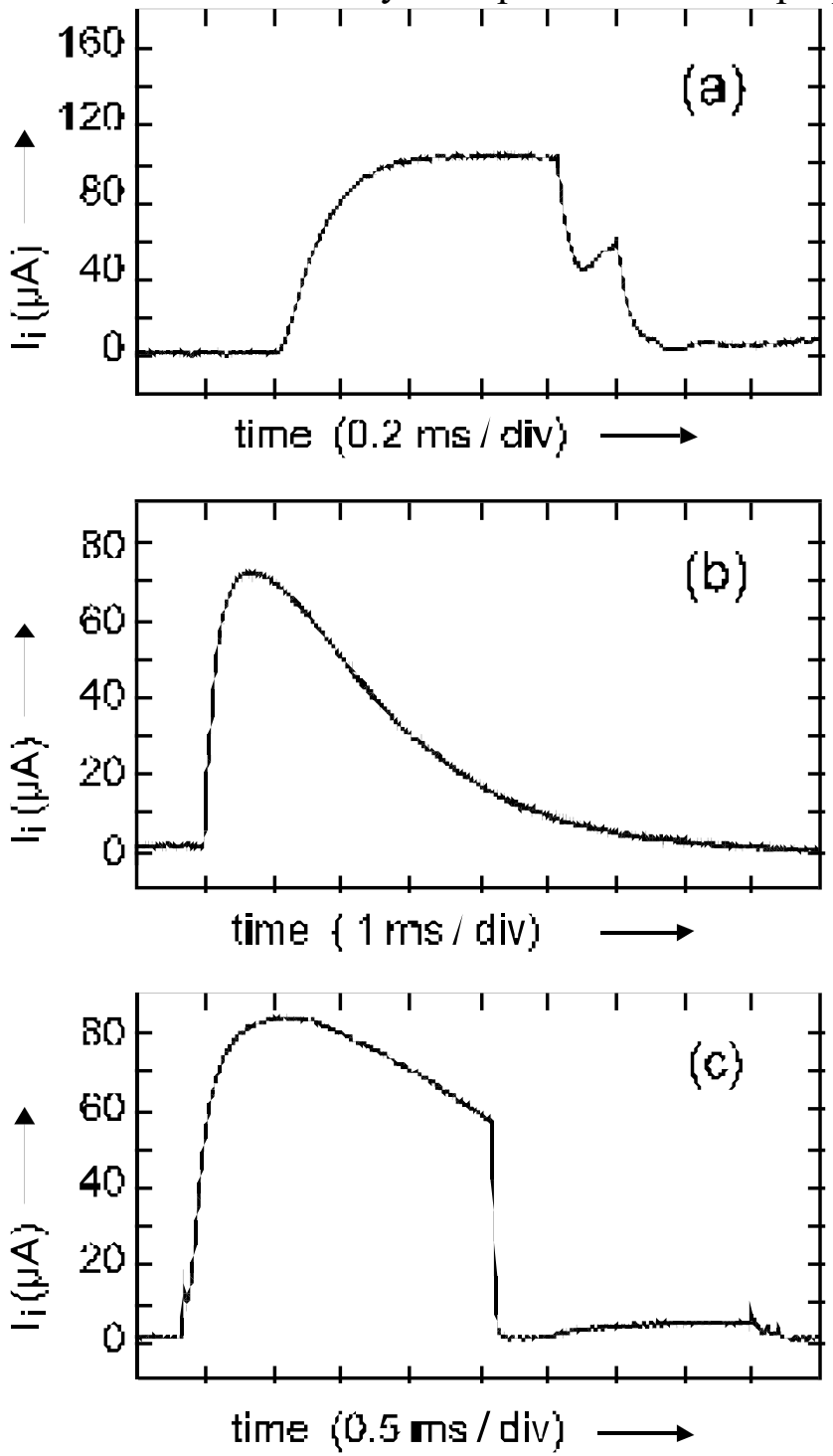

FIG. 3 Three different types of afterglow modes; a) Standard or "GANIL" mode; b) Stable mode; c) Operational Mode

\section{OPERATIONAL PROBLEMS}

In reality, few problems were experienced with the source. The only item of concern was a hypersensitivity of the microwave generator interlocks to outside perturbations. This problem is being dealt with on a case by case basis. The microoven also performed reliably and lead consumptions of between 5 and $7 \mathrm{mg}$ per day usually allowed a month between reloads of the sample. 
Cold starts of the source could be carried out in about two hours to the achievement of reasonable intensities. However, beam stability was generally not attained for about eight hours. Regular trimming of parameters enabled the performance to be maintained. Once into a stable regime, generally the only adjustments needed were in order to maintain the waveguide co-axial transition tuning at an optimum for beam intensity. This optimum was not at minimum reflected power but was probably that which produced a voltage anti-node at the plasma end of the line.

\section{OPERATIONAL PERFORMANCE}

The real test of the experience gained during the commissioning phase came when the Linac3 went on line for experimental physics. An eight week period from the middle of October 1994 had been allocated for this purpose. During this time the source provided $80 \mathrm{e} \mu \mathrm{A}$ of $\mathrm{Pb}^{27+}$, of which 50-60 e $\mu \mathrm{A}$ reached the stripper at 4.2 $\mathrm{MeV} / \mathrm{u}$. Figure 4a shows a typical charge state distribution found at the Faraday cup for normal operational settings of the source. After stripping and filtering, about 20 e $\mu \mathrm{A}$ of $\mathrm{Pb}^{53+}$ remained for further acceleration. This acceleration in the PS booster, PS, (stripping to $82+$ ) and of four PS pulses in the SPS resulted in $2.9 \times 10^{8}$ ions at $157 \mathrm{GeV} / \mathrm{u}$ being delivered to experiments ${ }^{5}$.

At the end of this period, beam was then supplied to the Low Energy Antiproton Ring (LEAR) at $4.2 \mathrm{MeV} / \mathrm{u}$ for beam cooling and lifetime experiments ${ }^{6}$. However, this experiment was unfortunately perturbed by microwave transmitter problems.

\section{IMPROVEMENTS}

During the commissioning and running period, the extraction gap of the source had been left at $42 \mathrm{~mm}$. Tests have since been carried out to determine the effect of variations of this gap. In each case the source (and the beam transport) was optimized to give the best stable beam at the Faraday cup as compared with the operational beam. The results for each gap were:-

$\begin{array}{ll}32 \mathrm{~mm} & 70 \mathrm{e \mu A} \\ 37 \mathrm{~mm} & 90 \mathrm{e} \mu \mathrm{A} \\ 42 \mathrm{~mm} & 110 \mathrm{e} \mu \mathrm{A} \\ 47 \mathrm{~mm} & 120 \mathrm{e \mu \textrm {A }}\end{array}$

At the smaller gaps, it proved very difficult to obtain and maintain the extraction voltage with magnetic field over all operating ranges without excessive discharges. However, the $47 \mathrm{~mm}$ gap has been retained for future operation. Further studies, both on the source itself and with simulations, are needed to see the limits of the extraction gap.

It had been suggested ${ }^{7}$ that the replacement of the oxygen pilot gas by neon should give rise to an improvement in intensity and an increase in mean charge state. 
Early tests indicated that, for $\mathrm{Pb}^{27+}$, this was not the case and oxygen was essential for optimum $27+$ production. More recent tests have shown that whereas there was no increase in intensity, the charge state distribution did shift to higher mean charge. Figure $4 \mathrm{~b}$ shows a charge state distribution in neon operation. $\mathrm{Pb}^{32+}$ was successfully extracted from the source at $20 \mathrm{kV}$ extraction voltage.
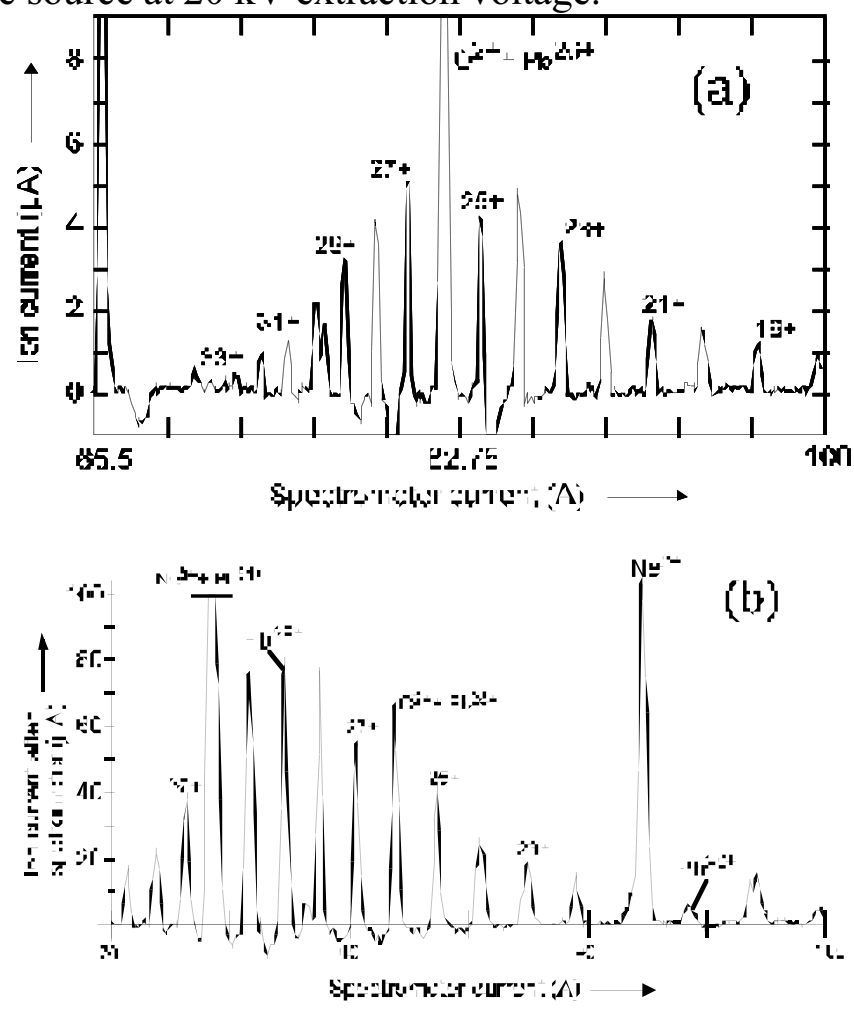

FIG. 4 Charge state distributions during the afterglow for: -a) Normal oxygen operation and b) Oxygen and neon as plasma gas

\section{FUTURE PLANS}

Further studies will need to be undertaken to examine the effects of the extraction gap on intensity and transmission of the beam to the RFQ. It has been observed that much higher intensities can be extracted but with a much reduced stability. A thorough search of the source parameter space will be required to try to exploit this intensity. Having now produced a lead beam some effort will be needed to examine methods of injecting other samples into the source to exploit the full range of heavy ions that could be requested by the high energy heavy ion physics community.

\section{ACKNOWLEDGMENTS}

The results achieved in running the source on the new Heavy Ion Linac would not have been possible without the development work on, and the delivery of the source by, the GANIL team (M-P. Bourgarel, M. Bisch, P. Leherissier, G. Schmiechen); nor would it have been possible to continue from their ground work without the assistance of CERN colleagues and services and especially H. Charmot and M. O'Neil. 


\section{REFERENCES}

1) D. Warner (Editor), CERN 93-01, CERN, Geneva (1993)

2) P. Briand, R. Geller, H. Haseroth, C. Hill, K. Langbein, Proc. 11t Int. Workshop on ECR Ion Sources, Groningen, KVI Report 996, 132 (1993)

3) M.P. Bourgarel, P. Sortais, P. Attal, M. Bisch, P.M. Cornen, P. Leherissier,J.Y. Pacquet, J.P. Rataud, G. Schmiechen, EPAC 90, Nice, Editions Frontières (1990)

4) M-P. Bourgarel, C.E. Hill, H. Haseroth, K. Langbein, E. Tanke, Proc. 12th Int. Workshop on ECR Ion Sources, Riken 1995. To be published.

5) H. Haseroth, 1985 Particle Accelerator Conference, Dallas 1995. To be published.

6) S. Baird, J. Bosser, M. Chanel, J. Duran, R. Giannini, P. Lefevre, R. Ley, R. Maccaferri, S. Maury, I. Meskov, D. Möhl, G. Molinari, F. Motsch, H. Mulder, U. Oftiger, J. Perrier, E. Roux, G. Tranquille, F. Varenne, CERN PA/AR/Note 95-6 (1995)

7) G. Shirkov, NIM, B, 98, 536 (1995) 